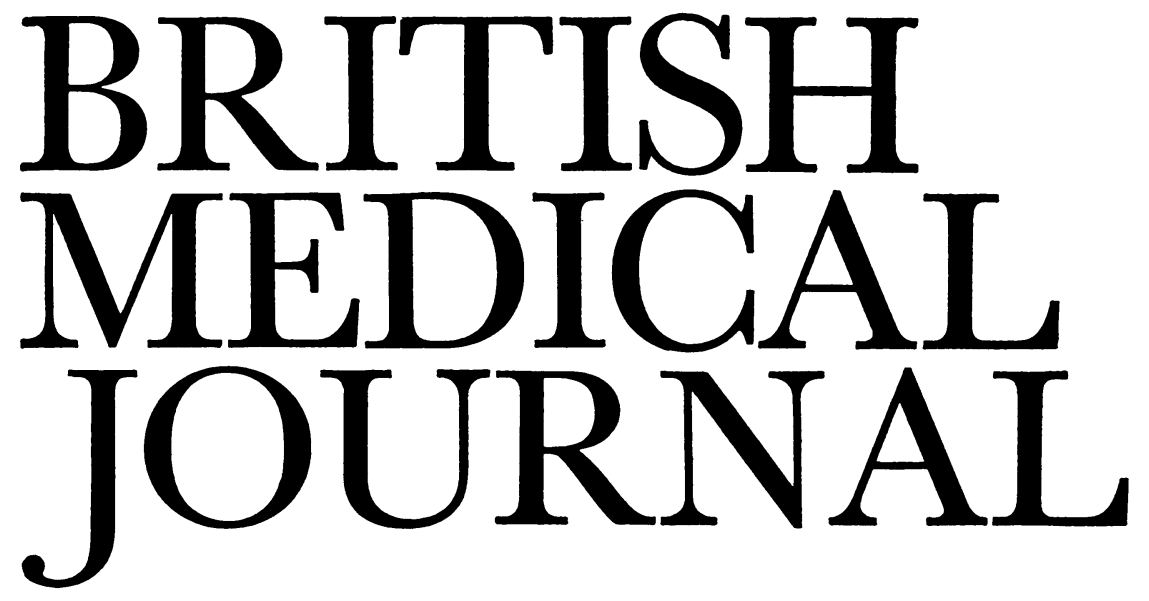

\title{
Levodopa: long-term impact on Parkinson's disease
}

Levodopa and its analogues have firmly established their place as symptomatic treatments for Parkinson's disease. Nevertheless, before dramatic claims can be made, their long-term benefits have to be compared with the natural course of the untreated disease.

Before levodopa, almost all patients were treated with belladonna alkaloids or synthetic anticholinergic drugs, and these may have modified the course of the illness. The brains of patients with Parkinson's disease show characteristic changes in the substantia nigra and also senile plaques, neurofibrillary tangles, and granulovacuolar degeneration indistinguishable from Alzheimer's disease. ${ }^{1}$ In Alzheimer's dementia cholinergic neurones are depleted, and treatment with choline, lecithin, and anticholinesterases has occasionally produced improvement in memory, cognition, and behaviour. ${ }^{2}$ Longterm anticholinergic treatment may have altered the prognosis of Parkinsonism in the era before levodopa, and we must not assume that data on these patients form an adequate control by which the effects of drugs can be judged.

Hoehn and Yahr ${ }^{3}$ reported 293 deaths among 672 patients followed for 15 years (1949-64) with a mean age at death of 67. The mean duration of illness was just under 10 years. Other workers claimed similar results, observing that patients had a mortality three times greater than controls. Morbidity, too, was high: a Scandinavian study ${ }^{4}$ showed that half of the patients became incapable of work four years after the onset of their disease. Clinical impression suggests the existence of subgroups: some have an early onset below the age of 40 , and another group have a late age of onset and may have a more benign prognosis. Survival for over 18 years has been shown in up to $25 \%$ of patients. ${ }^{5}$

How has levodopa altered these figures and, more important, the outlook for the individual ? The initial response is impressive in three-quarters of patients, with substantial improvement in the speed of movement, rigidity, and posture. Indeed, a lack of response should arouse the suspicion that the patient is not taking the tablets; that other drugs (pyridoxine in tonics, phenothiazines for dizziness or nausea) are interacting; or that some more generalised disorder (such as striatonigral degeneration or Shy-Drager syndrome) has been overlooked. Nevertheless, a few patients genuinely fail to respond and the mechanism of this anomaly is being studied.

After two years of treatment Stern and his colleagues ${ }^{6}$ found that only $60 \%$ of patients had maintained the initial level of improvement and that complications of the drug had steadily increased. Two common problems were dyskinetic movements and on-off swings in performance, related to individual doses of drugs and also occurring sporadically irrespective of both the time of the dosage and blood concentrations. Transient hallucinations, nightmares, and frank psychoses increased in frequency and sometimes became serious problems after the first year or two of levodopa. Many patients, however, continued to benefit substantially and were capable of independent productive activities both at home and at work at this stage.

Shaw, Lees, and Stern have now published a comprehensive review of 178 patients followed up for six years. ${ }^{7}$ Some $70 \%$ showed an initial response to levodopa in the "marked to moderate" range, with a mean $50 \%$ reduction in disability. After two years 62 patients had dropped out because of side effects or because their response was inadequate; $63 \%$ of patients receiving treatment still maintained a response better than $50 \%$. After six years half the patients had dropped out, but of those 81 still receiving treatment with levodopa 36 had a "moderate" and one a "marked" response; 47 of the 178 were still less disabled than at the onset. The ratio of observed to expected death rate for all the patients was $1 \cdot 45: 1$. In those patients who were unable to tolerate levodopa for longer than two years the ratio was $2 \cdot 38: 1$, whereas in those who could tolerate sustained medication life expectancy was normal.

Psychiatric complications are the most serious side effects of levodopa, often necessitating reduction or withdrawal of treatment. Transient toxic confusional states with nightmares and hallucinations may be initially controllable by adjustment of dosage and by simple hypnotics. Visual hallucinations increase as treatment continues, occurring by night or day. Depression is found in $22 \%$ of patients ${ }^{7}$ before treatment and is sometimes improved along with the physical benefits when levodopa is first given. After six years, however, a quarter of the patients appear moderately or severely depressed. Combinations of insomnia, confusional episodes, and depression lead to a mixed and fluctuating psychotic state. Dementia may develop in time, and is conspicuous in a third of patients after six years. Since there is evidence of increased survival with treatment, it may be argued that some of the demented patients have simply lived longer and are, therefore, prone to other dementing processes which accompany aging. Cortical atrophy and ventricular dilatation shown on CT scans are common in these patients, but again may simply reflect aging. Alternatively, 
the atrophy and dementia might possibly be precipitated prematurely by dopaminergic or anticholinergic drugs. Accumulated experience suggests that giving the maximum tolerated dosage of dopamine replacement may be injudicious until we can be more certain that this does not accelerate both dementia and brain atrophy.

Bromocriptine, a dopamine agonist, is an effective if expensive alternative treatment. ${ }^{8}$ Its side effects are, however, similar to those of levodopa. When bromocriptine is given with small doses of levodopa the combination seems able to prolong the total useful period of active treatment.

Where, then, do we stand ? For between one-third and a half of the clinical course of the disease many patients will benefit greatly from treatment with dopamine replacements or dopamine agonists. The start of such treatment should be delayed until the patient is beginning to find difficulty in coping with housework, shopping, or work. Probably the dosage should be kept as low as is consistent with achieving good functional improvement, particularly at the onset of treatment. Nevertheless, with all their limitations these drugs improve the quality of life and probably prolong survival to about 14 years from the onset of the disease.

${ }^{1}$ Hakim AM, Mathieson G. Dementia in Parkinson's disease: a neuropathologic study. Neurology 1979;29:1209-14.

2 Etienne P, Gauthier S, Johnson G, et al. Clinical effects of choline in Alzheimer's disease. Lancet 1978;i:508-9.

${ }^{3}$ Hoehn MM, Yahr MD. Parkinsonism: onset, progression, and mortality. Neurology (Minneap) 1967;17:427-42.

${ }^{4}$ Mjönes H. Paralysis agitans: a clinical and genetic study. Acta Psychiatrica et Neurologica Scandinavica 1949;54:1-195.

5 Pollock M, Hornabrook RW. The prevalence, natural history and dementia of Parkinson's disease. Brain 1966;89:429-48.

${ }^{6}$ Hunter KR, Shaw KM, Laurence DR, Stern GM. Sustained levodopa therapy in Parkinsonism. Lancet 1973;ii:929-31.

7 Shaw KM, Lees AJ, Stern GM. The impact of treatment with levodopa on Parkinson's disease. Q $\mathcal{F}$ Med 1980;49:283-93.

${ }^{8}$ Pearce JMS. Aetiology and natural history of Parkinson's disease. $\mathrm{Br} \mathrm{Med} \mathcal{F}$ 1978;ii:1664-6.

\section{Prostaglandins in obstetrics}

Prostaglandin preparations have now been used for over a decade for terminating pregnancy and for inducing labour, ${ }^{1}$ particularly when the cervix is unfavourable. ${ }^{2}{ }^{3}$ More recently pretreatment with prostaglandin has come into favour as a means of making suction termination of pregnancy less hazardous for nulliparous young women: the softening of the cervix makes dilatation easier and less traumatic.

Prostaglandins might have been given to more patients had they not had two disadvantages: a high incidence of distressing side effects and a relatively unpredictable action. In an attempt to overcome these problems many different routes of administration have been tried, including intra-amniotic, extraamniotic, intravenous, and oral and-less commonlyintramuscular injections, vaginal pessaries, and cervical gels.

The intra-amniotic route has proved excellent for the induction of midtrimester abortion. ${ }^{4}$ Slow absorption from the amniotic cavity keeps to a minimum the frequency and severity of side effects, but repeated doses of prostaglandin $E_{2}$ or $F_{2 \alpha}$ are often needed to achieve a success rate of $95 \%$. The 15-methyl analogues were developed in an attempt to improve the potency and prolong the duration of action of prostaglandins $E_{2}$ and $F_{2 \alpha}{ }^{5}$; these are resistant to degradation by the enzyme 15-hydroxy dehydrogenase. Clinical trials of 15-methyl prostaglandins using different routes have not always confirmed the initial expectations, ${ }^{67}$ but in 1973 Amy et $a l^{8}$ reported successful midtrimester termination of pregnancy in 18 out of 20 patients who were given a single $100 \mu \mathrm{g}$ dose of the 15-methyl $\mathrm{E}_{2}$ analogue by intra-amniotic injection. Side effects were minimal, but an attempt to reduce the induction-abortion interval from 16.5 hours by doubling the dose was unsuccessful. The increased dose also increases the risk of serious cardiovascular or respiratory side effects if the drug is absorbed unusually rapidly or accidentally injected intravenously.

The extra-amniotic routes of administration of prostaglandins were first described by Wiqvist and Bygdeman in $1970 .{ }^{9}$ Reports have shown some variation in the inductionabortion interval but it has generally been below 36 hours. The disadvantage of the need for repeated instillation of the drug through a transcervical catheter has been largely overcome by the introduction of continuous infusion systems. Concern that the presence of a catheter in the uterus would result in an increased risk of infection has generally been unfounded, but the possibility needs to be taken into account when choosing this method.

From the patients' standpoint the most convenient and acceptable preparation is a tablet. The many clinical trials ${ }^{10-12}$ of oral prostaglandins in inducing labour and terminating pregnancy have given consistent results. Labour can be induced successfully by this technique and the results are comparable with those of induction by intravenous oxytocin. Nevertheless, repeated doses are required and the best regimen remains to be determined. Bremme et $a l^{13}$ recently reported a series of 30 women in whom labour was induced using oral prostaglandin $\mathrm{E}_{2}$ when pregnancy was near full term. Three different dose schedules were evaluated, and they found that a dose of $1 \mathrm{mg}$ hourly will result in a stable plasma concentration with little evidence of accumulation and consequently less risk of uterine hyperstimulation.

Another new method recently developed for the introduction of prostaglandins is by intravaginal or intracervical gel.14 Incremental doses may be necessary to establish progressive labour, and a major disadvantage is that there is no means of control of the rate of absorption.

No doubt remains about the efficacy of prostaglandins in the induction of labour or therapeutic abortion, but there remain the problems associated with the incidence of doserelated side effects and with unpredictable oxytocic activity. Predicting the pharmacological effects of the agent depends especially on achieving a constant rate of absorption, and if this could be achieved for a convenient, acceptable preparation of prostaglandin its undoubted therapeutic value would be increased.

${ }^{1}$ Beazley JM, Dewhurst CJ, Gillespie A. The induction of labour with prostaglandin $\mathrm{E}_{2}$. Fournal of Obstetrics and Gynaecology of the British Commonwealth 1970;77:193-9.

2 MacKenzie IZ, Embrey MP. Cervical ripening with intravaginal prostaglandin $\mathrm{E}_{2}$ gel. Br Med $\mathcal{F} 1977 ; \mathrm{ii}: 1381-4$.

3 Hutchon DJR, Geirsson R, Patel NB. Int $\mathcal{f}$ Gynaecol Obstet 1980;17: 604-7.

4 Southern EM. Prostaglandins: clinical applications in human reproduction. New York: Futura Publishing Co, 1972.

5 Karim SMM, Sharma SD. Termination of second trimester pregnancy with 15 methyl analogues of prostaglandins $E_{2}$ and $F_{2} \alpha$. fournal of Obstetrics and Gynaecology of the British Commonwealth 1972;79: 737-43.

6 Karim SMM, Sivasamboo R. Termination of second trimester pregnancy with intra-amniotic 15 (S) 15 methyl prostaglandin F-2alpha-a two dose schedule study. Prostaglandins 1975;9:487-94.

7 Krishna U, Ganguli AC, MandlekarAV, Purandare VN. Administration of prostaglandins by various routes for induction of abortion. Prostaglandins 1978;15:685-93. 